\title{
Heating of opaque surfaces by picosecond laser pulses
}

\section{H. Strehlow}

Max-Planck-Institut für Biophysikalische Chemie, 37018 Göttingen, Germany

Received: 5 June 1997/Accepted: 13 June 1997

\begin{abstract}
Heat conduction after the application of a short laser pulse on opaque matter is calculated assuming simplifying conditions. The effect of the simplifications on real systems is discussed. It is found that Fourier's law may reasonably be applied considerably above the temperature of vaporization of the substrate. Calculated and experimental ablation at nickel surfaces using fluences below threshold are compared.
\end{abstract}

PACS: $65 ; 81.60$

In laser processing technology the problem of energy dissipation is of paramount importance [1-3]. When working with short laser pulses on matter with high extinction coefficients and high thermal diffusivity (metals and semiconductors) heat conduction cannot be neglected even when the picosecond laser pulse has not yet decayed to zero. In the next section a simplified model for heat conduction under these conditions will be derived. A closed solution of Fourier's differential equation will be presented. The simplifying assumptions are: 1. Heat flow will be understood to be restricted to one dimension.

2. The physical parameters (thermal diffusivity, density, absorption coefficient, specific heat, reflectivity) will be assumed to be independent of temperature.

3. The form of the laser pulse is given by (1b) below.

4. The effect of the enthalpy of phase changes is neglected.

5. Only heat conduction is assumed to be effective in energy transport. Stefan-Boltzmann radiation is neglected

6. Also ablation of matter at very high temperatures is not considered.

Section 2 will discuss the effect of these simplifications when applying the solution of the model to real systems.

\section{Fourier's equation}

For an irradiated substrate with an optical extinction coefficient $\alpha$ Fourier's equation in one dimension reads:

$$
\frac{\partial \theta}{\partial t}=\kappa \frac{\partial^{2} \theta}{\partial x^{2}}+F(t) \exp (-\alpha x)
$$

where $\theta(x, t)=T(x, t)-T(x, 0)$ and $F(t)$ is the pulse form of the laser flash. $\kappa$ is the thermal diffusivity. The initial and boundary conditions are:

$\theta(x, 0)=0$ and $\theta(\infty, t)=0$.

The problem, that according to (1) with large negative values of $x$ the energy input diverges, will be solved below by using appropriate integration constants.

For short picosecond pulses a pulse form $F(t)$ is assumed:

$F(t)=A\left(\frac{t}{\tau}\right) \exp \left(\frac{-t}{\tau}\right)$.

(The half-width of this pulse amounts to $2.795 \cdot \tau$.) Then differential equation (1) reads:

$\frac{\partial \theta}{\partial t}=\kappa \frac{\partial^{2} \theta}{\partial x^{2}}+A\left(\frac{t}{\tau}\right) \exp \left(\frac{-t}{\tau}\right) \exp (-\alpha x)$.

Applying a Laplace transformation on the partial differential equation (1c) leads to the ordinary differential equation:

$\frac{\mathrm{d}^{2} \Theta}{\mathrm{d} x^{2}}-k^{2} \Theta=-\frac{A \tau \exp (-\alpha x)}{\kappa(1+\tau s)^{2}}$

with

$k^{2}=\frac{s}{\kappa}$.

For $\Theta(x, s)$, the Laplace transform of $\theta(x, t)$, the solution is:

$\Theta=C_{1} \exp (-k x)+C_{2} \exp (k x)+\Theta_{\mathrm{p}}$

where the first two terms are the general and the last term is the particular integral. $s$ is the Laplace parameter and $C_{i}$ are the integration constants to be determined using the boundary and initial conditions. Since for large values of $x, \theta(x, t)=0$ (and therefore $\Theta(x, s)=0), C_{2}$ must be zero. $C_{1}$ is only a constant with respect to $x$ but may depend on the parameter $s . C_{1}$ will be determined after the particular integral $\Theta_{\mathrm{p}}$ has been calculated. With:

$B=\frac{-A \tau}{\kappa(1+\tau s)^{2}}$ 
the solution for $\Theta_{\mathrm{p}}$ is:

$$
\begin{aligned}
\Theta_{\mathrm{p}} & =B \exp (-k x) \int \exp (2 k x)\left[\int \exp (-k x)\right. \\
& =\frac{B \exp (-\alpha x)}{k^{2}-\alpha^{2}}=\frac{B \exp (-\alpha x)}{(k-\alpha)(k+\alpha)}
\end{aligned}
$$

and

$\Theta=C_{1}(s) \exp (-k x)+\frac{B \exp (-\alpha x)}{(k-\alpha)(k+\alpha)}$.

After back transformation the second term on the right-hand side of (6) diverges for large $t$ [see Table 1: $f_{0}(s)$ ]. The physical reason is the heat flow from the infinite heat reservoir with $x<0$. By a suitable choice of $C_{1}(s)$ the heat flow from $x<0$ can be made to vanish and the divergence of (6) at large $t$ disappears. This is achieved by:

$C_{1}(s)=\frac{B \alpha}{(k+\alpha) k^{2}}$

Resubstitution of $C_{1}(s)$ from (7) as well as $B$ and $k$ from (4) and (2a) into (6) result in:

$\Theta=\frac{-a A}{(s+a)^{2}}\left[\frac{b \exp (-c \sqrt{s})}{s(\sqrt{s}+b)}+\frac{\exp (-\alpha x)}{(\sqrt{s}-b)(\sqrt{s}+b)}\right]$

with

$a=\frac{1}{\tau}, \quad b=\alpha \sqrt{\kappa}, \quad$ and $\quad c=\frac{x}{\sqrt{\kappa}}$.

To avoid heat flow from the region with $x<0$ the gradient of $\Theta$ at $x=0$ is set equal to zero. (If at $x=0$ no heat flows from $x<0$ but does so in the positive $x$ direction, the gradient

Table 1. Laplace transform pairs

\begin{tabular}{ll}
\hline$f(s)$ & $F(t)$ \\
\hline$f_{0}(s)=\frac{1}{s-b^{2}}$ & $F_{0}(t)=\exp \left(b^{2} t\right)$ \\
$f_{1}(s)=\frac{1}{s+a^{2}}$ & $F_{1}(t)=t \exp (-a t)$ \\
$f_{2}(s)=\frac{1}{s}$ & $F_{2}(t)=1$ \\
$f_{12}(s)=f_{1}(s) f_{2}(s)$ & $F_{12}(t)=F_{1}(t) * F_{2}(t)=\frac{1-(a t+1) \exp (-a t)}{a^{2}}$ \\
$f_{3}(s)=\frac{1}{\sqrt{s}(\sqrt{s}+b)}$ & $F_{3}(t)=\exp \left(b^{2} t\right) \operatorname{erfc}(b \sqrt{t})$ \\
$f_{4}(s)=\frac{\exp (-c \sqrt{s})}{\sqrt{s}+b}$ & $F_{4}(t)=\frac{1}{\sqrt{\pi t}} \exp \left(-\frac{c^{2}}{4 t}\right)$ \\
& $-b \exp (b c) \exp \left(b^{2} t\right) \operatorname{erfc}\left(b \sqrt{t}+\frac{c}{2 \sqrt{t}}\right)$
\end{tabular}

becomes zero.)

$$
\begin{aligned}
\left(\frac{\mathrm{d} \Theta}{\mathrm{d} x}\right)_{x=0}=\frac{-a A}{(s+a)^{2}} & {\left[\frac{-\alpha}{\sqrt{s}(\sqrt{s}+b)}\right.} \\
& \left.-\frac{\alpha}{(\sqrt{s}-b)(\sqrt{s}+b)}\right]=0 .
\end{aligned}
$$

With (10) the factor responsible for the divergence is eliminated:

$\frac{1}{\sqrt{s}-b}=-\frac{1}{\sqrt{s}}$

and

$\Theta=\frac{A a}{(s+a)^{2}}\left[\frac{\exp (-\alpha x)}{\sqrt{s}(\sqrt{s}+b)}-\frac{b \exp (-c \sqrt{s})}{s(\sqrt{s}+b)}\right]$.

For these functions $f(s)$ the Laplace retransformations are not available from the tables. However, convolution allows us to retransform a product, provided the retransforms of the factors $F_{i}(t)$ are known. The convolution theorem reads:

$\mathrm{L}^{-1} f_{i}(s) f_{k}(s)=\int_{0}^{t} F_{i}(t-z) F_{k}(z) \mathrm{d} z \equiv F_{i} * F_{k}$

where $L^{-1}$ is the inverse Laplace operator. The result is then:

$\theta(t, x)=A\left[F_{1} * F_{3} \exp (-\alpha x)-b F_{12} * F_{4}\right]$.

Table 1 contains the relevant transform pairs.

The convolution $F_{1} * F_{3}$ may be executed by partial integration of the convolution integral and using a series development that is more precise and faster than an integration using Simpson's method. We thus obtain, after resubstituting $a, b$, and $c$ [see (9)]:

$$
\begin{aligned}
F_{1} * F_{3}= & \frac{\tau^{2}}{\left(1+\alpha^{2} \kappa \tau\right)^{2}} \exp \left(\alpha^{2} \kappa t\right) \operatorname{erfc}(\alpha \sqrt{\kappa t}) \\
& +\frac{\tau \exp (-t / \tau)}{1+\alpha^{2} \kappa \tau}\left[\frac{\tau \alpha \sqrt{\kappa t}}{\left(1+\alpha^{2} \kappa \tau\right) \sqrt{\pi}} \sum_{i=0}^{\infty} \frac{2(t / \tau)^{i}}{i !(2 i+1)}\right. \\
& \left.-\frac{\tau}{1+\alpha^{2} \kappa \tau}-t+\frac{\alpha t \sqrt{\kappa t}}{\sqrt{\pi}} \sum_{i=0}^{\infty} \frac{4(t / \tau)^{i}}{i !(2 i+1)(2 i+3)}\right] .
\end{aligned}
$$

The convolution $F_{12} * F_{4}$ will be performed using Simpson's rule:

$$
\begin{aligned}
F_{12} * F_{4}= & \tau^{2} \int_{0}^{t}\left\{1-\left[\frac{(t-z)}{\tau}+1\right] \exp \left[-\frac{(t-z)}{\tau}\right]\right\} \\
\times & \left\{\frac{\exp \left(-x^{2} / 4 \kappa z\right)}{\sqrt{\pi z}}-\alpha \sqrt{\kappa} \exp (\alpha x) \exp \left(\alpha^{2} \kappa z\right)\right. \\
& \left.\operatorname{erfc}\left[\alpha \sqrt{\kappa z}+\frac{x}{2 \sqrt{\kappa z}}\right]\right\} \mathrm{d} z
\end{aligned}
$$




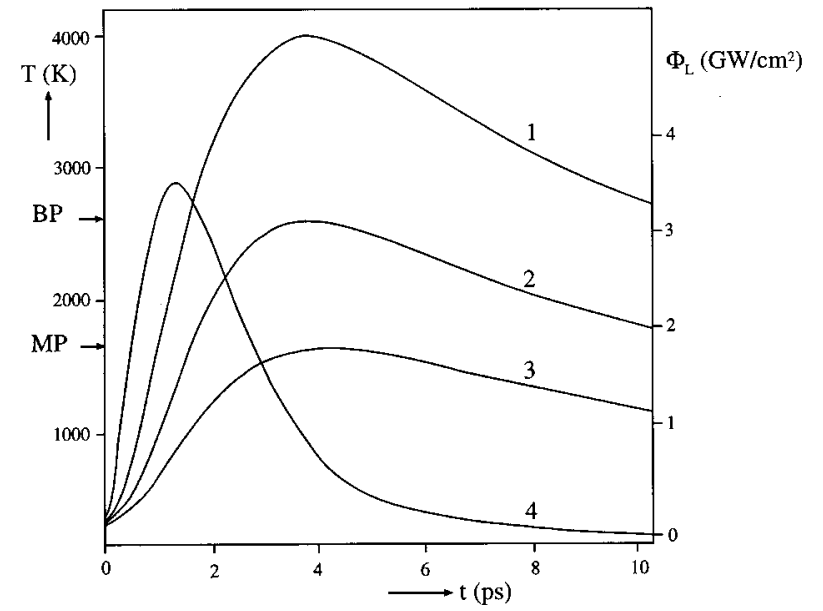

Fig. 1. Temperature against time $t$ after applying a laser pulse to silicon. Temperature independent parameters: applied energy per area $(1-R) \phi: 10 \mathrm{~mJ} / \mathrm{cm}^{2}$; time constant of the laser pulse: $\tau=1 \mathrm{ps}$; thermal diffusivity $\kappa=0.145 \mathrm{~cm}^{2} / \mathrm{s}$; specific heat $C=0.71 \mathrm{~J} / \mathrm{gK}$; density $\varrho=2.32 \mathrm{~g} / \mathrm{cm}^{3}$; extinction coefficient $\alpha=10^{6} \mathrm{~cm}^{-1}$. Curve $1: x=0$, curve 2: $x=5$, curve 3: $x=10 \mathrm{~nm}$, curve 4: power of the laser pulse; right ordinate. MP: melting point $(1690 \mathrm{~K}), \mathrm{BP}$ : boiling point of silicon $(2628 \mathrm{~K})$

( $F_{12} * F_{4}$ decays to zero with time much faster than does $F_{1} * F_{3}$ and the error arising by using Simpson's method is less serious.)

Finally the dependence of the constant $A$ in (14) on the physical parameters of the system must be calculated. For $t \gg \tau$ the integral of $\theta$ over $x$ is constant, since no further energy is supplied or lost according to the assumptions listed above before Sect. 1. The input fluence (= energy input per area) $(1-R) \phi$, calculated for the case of vanishing thermal diffusivity $(\kappa \rightarrow 0)$ is given by:

$$
(1-R) \phi=C \varrho \int_{0}^{\infty} \int_{0}^{\infty} A\left(\frac{t}{\tau}\right) \exp \left(\frac{-t}{\tau}\right) \exp (-\alpha x) \mathrm{d} t \mathrm{~d} x
$$

where $R$ is the reflectivity, $\varrho$ is the density $\left(\mathrm{g} / \mathrm{cm}^{3}\right), \alpha$ the absorption coefficient $\left(\mathrm{nm}^{-1}\right), \tau$ is the time constant of the laser pulse (ps), and $C$ is the specific heat $(\mathrm{J} / \mathrm{gK})$. (Under the conditions at issue the specific heat $C$ is neither at constant pressure nor at constant volume. However, the difference is only marginal and may safely be neglected.) Since the total energy input does not depend on the value of $\kappa,(17)$ is also valid for $\kappa \neq 0$. If $\phi$ is in $\mathrm{mJ} / \mathrm{cm}^{2}$ from (17) with the chosen units $A$ is given by:

$A=\frac{10^{4} \alpha(1-R) \phi}{C \varrho \tau}$.

Combining (14), (15), (16) and (18) supplies the dependence of the temperature as a function of $t$ and $x$ under the restricting conditions listed above. Figure 1 shows $\theta(t, x)$ as a function of $t$ (with $x$ as a parameter). The data are calculated for silicon as the substrate under the conditions listed before Sect. 1.

The dependence of the thermal diffusivity and of the specific heat of silicon on temperature has been obtained by Shanks et al. [4]. $\kappa$ decreases from $200 \mathrm{~K}$ to $800 \mathrm{~K}$ from
$0.9 \mathrm{~cm}^{2} \mathrm{~s}^{-1}$ to $0.145 \mathrm{~cm}^{2} \mathrm{~s}^{-1}$ and remains at this value at higher temperatures. The temperature coefficient of the density has been taken from [5]. The influence of these dependencies on heat conduction will be discussed in Sect. 2.2. See Fig. 2.

\section{Real and idealized heat conduction systems}

In this section the consequences of the simplifications listed before Sect. 1 for the evaluation of $\theta(t, x)$ for real physical systems will be estimated at least approximately.

\subsection{Heat flow in one dimension}

A focused laser beam entering the substrate in the $x$ direction will not be homogeneous in the $y$ and $z$ directions. The energy flux entering the substrate at $x=0$ can be described by a Gaussian curve:

$F(y, z)=F(0,0) \exp \left[-2 \frac{\left(y^{2}+z^{2}\right)}{\sigma^{2}}\right]$.

Since we are dealing with opaque materials with the thickness of the heated material $1 / \alpha$ on the order of $10 \mathrm{~nm}$ and since $\sigma$ will be at least on the order of $\mu \mathrm{m}$, the temperature gradients in the $y$ and $z$ directions are considerably smaller than the gradient in the $x$ direction. Under these conditions we can neglect the heat flow in both the $y$ and the $z$ directions. Then $\theta(t, x, y, z)$ can be written:

$\theta(t, x, y, z)=\theta(t, x, 0,0) \exp \left[-2 \frac{\left(y^{2}+z^{2}\right)}{\sigma^{2}}\right]$.

\subsection{Temperature dependence of the parameters}

Let $P_{k}$ be any relevant parameter such as reflectivity, absorption coefficient, specific heat, or density. (For thermal diffusivity see below.) All $P_{k}$ have been assumed to be independent of temperature in the treatment of Sect. 1. Unfortunately, not many data are reported for $P_{k}(T)$ but the few known data do not indicate a dramatic variance of the $P_{k}$ with temperature. Due to the high temperatures involved, however, the effects of the temperature dependencies of $P_{k}$ are in general not negligible.

If reliable data for $P_{k}(T)$ are available, a good approximate calculation of $\theta(t, x)$ is not difficult. The computer program calculates for equidistant times $t_{n}$ and also equidistant values $x_{\mathrm{m}}$ the temperatures $T_{n, m}=\theta\left(t_{n}, x_{\mathrm{m}}\right)+T_{0}$, with $T_{0}$ the ambient temperature. Starting with $n=1$ and $m=1$ and $T_{0, m}=T_{0}$ one sets $P_{k}\left(T_{n, m}\right)=P_{k}\left(T_{n-1, m}\right)$ into the program and calculates $T_{n, m}$ and this for all values of $n$ and $m$. This requires only a trivial modification of the computer program. Using large numbers $n$, the approximation will be good.

This procedure will not work that simply with a thermal diffusivity $\kappa$ depending on temperature (and therefore on $x$ and $t$ ). Then (1c) must be replaced by:

$$
\begin{aligned}
\frac{\partial \theta}{\partial t}= & \kappa(t, x) \frac{\partial^{2} \theta}{\partial x^{2}}+\frac{\partial \kappa(t, x)}{\partial x} \frac{\partial \theta}{\partial x} \\
& +A\left(\frac{t}{\tau}\right) \exp \left(\frac{-t}{\tau}\right) \exp (-\alpha x)
\end{aligned}
$$




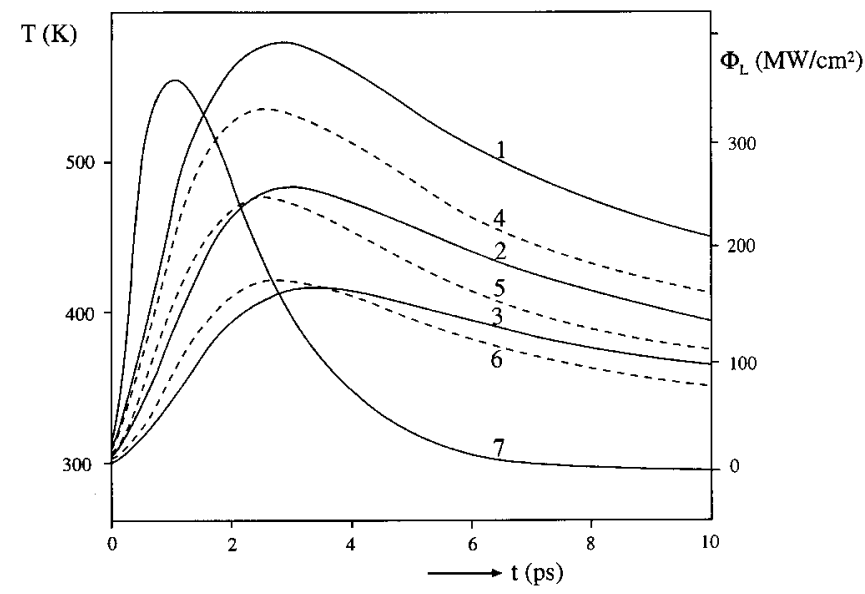

Fig. 2. Temperature against time $t$ after applying a laser pulse to silicon. Applied energy per area: $1 \mathrm{~mJ} / \mathrm{cm}^{2}$; time constant $T$ of the laser pulse: 1 ps. Curve 1, 2, and 3: average values in the temperature interval for the parameters have been used. Thermal diffusivity $\kappa=0.4 \mathrm{~cm}^{2} / \mathrm{s}$; specific heat $C=0.82 \mathrm{~J} / \mathrm{gK}$; density $\varrho=2.31 \mathrm{~g} / \mathrm{cm}^{3}$; extinction coefficient $\alpha=1.0 \times 10^{6} \mathrm{~cm}^{-1}$. Curve $4,5,6$ (dashed lines): temperature dependence of $\kappa$ [4], and $\varrho C[4,5]: \kappa=3.1931 \cdot \exp (-0.004872 \cdot T)+0.145$; $\varrho C=2.174-105.9 / T-14990 / T^{2}$. Other parameters as in curves 1 to 3 . Curves 1 and 4: $x=0$, curves 2 and 5: $x=5$, curves 3 and $6: x=10 \mathrm{~nm}$; curve 7: power of the laser pulse; right ordinate

With silicon as substrate, $\kappa$ does not vary with temperature above about $800 \mathrm{~K}$ [4]. However, at lower laser power the temperature remains below $800 \mathrm{~K}$, and the variation of $\kappa$ with temperature must be taken into account. An approximate estimation is obtained as follows. As a rather good approximation the dependence of $\theta(t, x)$ with $x$ is given by:

$\theta(t, x) \approx \theta(t, 0) \exp (-\alpha x)$

Under these conditions:

$$
\begin{aligned}
\frac{\partial \theta}{\partial x} & =-\theta(t, 0) \alpha \exp (-\alpha x) \quad \text { and } \\
\frac{\partial^{2} \theta}{\partial x^{2}} & =\theta(t, 0) \alpha^{2} \exp (-\alpha)
\end{aligned}
$$

Thus (1d) may be written:

$$
\frac{\partial \theta}{\partial t}=\tilde{\kappa} \frac{\partial^{2} \theta}{\partial x^{2}}+A\left(\frac{t}{\tau}\right) \exp \left(\frac{-t}{\tau}\right) \exp (-\alpha x)
$$

with

$\tilde{\kappa}=\kappa(T)\left[1+\frac{\partial \ln \kappa}{\partial T} \theta(t, 0) \exp (-\alpha x)\right]$.

In Fig. 2, $T(t, x)$ is shown for a low laser intensity $\left(1 \mathrm{~mJ} / \mathrm{cm}^{2}\right)$ with constant parameters averaged for the temperature interval, and $T(t, x)$ calculated using fitted experimental values for the specific heat [4], the density [5], and the thermal diffusivity [4] as a function of temperature. The temperature dependence of the absorption coefficient $\alpha$ is not known and $\alpha$ has been taken as a constant.

It should be realized that (1e) and (23) are only approximate. However, it is unlikely that the error thus introduced will be larger than that due to the uncertainty of experimental data.

\subsection{The form of the laser pulse}

The special pulse form

$F(t)=A\left(\frac{t}{\tau}\right) \exp \left(\frac{-t}{\tau}\right)$ for $t>0$

is a reasonable choice for picosecond laser pulses. The absorbed light energy at first excites electrons into higher energy states that relax exponentially in time dissipating their excitation energy into heat $[6,7]$. Thus the effective time constant $\tau$ in (1b) will be larger than the proper time constant $\tau_{\mathrm{L}}$ of the laser pulse, if the energy relaxation time $\tau_{\mathrm{e}}$ is comparable or even longer than $\tau_{\mathrm{L}}\left(\tau_{\mathrm{e}} \approx 0.5 \mathrm{ps}\right.$ [7]). The heat inflow will then follow a time law similar to (1b).

For laser pulses in the nanosecond range, (1b) might be a less useful approximation. The envelope of the series of short pulses constituting the ns-pulse in general has a form different from (1b); see [1].

\subsection{The effect of phase changes on heat conduction}

One might assume at first sight that the equations derived above would be applicable only up to the boiling point of the material at issue. This, however, must not be true in the picosecond time range. If the kinetics of evaporation and of melting is slow compared with the heating and heat flow process, the system will behave like a superheated solid. The heat conduction equation may then be applied to higher temperatures than the boiling point.

According to Knudsen [8] the rate of evaporation at temperature $T$ must be equal to the rate of deposition of atoms from the gas at equilibrium vapour pressure $p_{\mathrm{S}}(T)$. The latter rate can be calculated as the product of the concentration of the atoms in the gas phase and their mean velocity in the positive $x$ direction. This together with the ideal gas law leads to:

$j_{\mathrm{ev}}=\frac{\alpha_{\mathrm{ev}}\left(p_{\mathrm{s}}-p\right)}{\sqrt{2 \pi M R T}}$

where $\alpha_{\mathrm{ev}} \leq 1$ is the evaporation coefficient ( $\alpha_{\mathrm{ev}}=0.8$ for silicon), $R$ is the gas constant and $M$ the molecular (atomic) weight of the gas particles, $p_{\mathrm{s}}$ is the saturation pressure of the gas at the temperature $T$ and $p$ the actual pressure (that in case of evaporation into vacuum is zero). $j_{\text {ev }}$, the flux of evaporation, is given in $\mathrm{mol} / \mathrm{cm}^{2} \mathrm{~s}$ by (24). The heat flux due to evaporation (in $\mathrm{W} / \mathrm{cm}^{2}$ ) is then:

$-\Phi_{\mathrm{ev}}=j_{\mathrm{ev}}\left[\theta(t, 0) M C+\Delta H_{\mathrm{ev}}\right]$.

$\Delta H_{\mathrm{ev}}$ is the molar enthalpy of evaporation; $C$ is the specific heat, and $\theta(t, 0)=T-T_{0}$ is the temperature increase on the surface of the substrate (with $T_{0}$ the ambient temperature). This heat flux will now be compared with the flux due to heat conduction. A reasonable estimate for this flux is given by (first law of Fourier):

$-\Phi_{\mathrm{HF}}=\kappa \varrho C \cdot \operatorname{grad} T \approx \kappa \varrho C\left(T-T_{0}\right) \alpha$.

Pressures at temperatures above the boiling point of the substrate may be calculated using the Clausius-Clapeyron equation. (The temperature dependence of $\Delta H_{\mathrm{ev}}$ [3] is neglected. 
Table 2. Heat conduction and evaporation kinetics at silicon surfaces: Knudsen evaporation coefficient $\alpha_{\mathrm{ev}}=0.8$; thermal diffusivity $\kappa=$ $0.145 \mathrm{~cm}^{2} \mathrm{~s}^{-1}$; density $\varrho=2.33 \mathrm{~g} \mathrm{~cm}^{-3}$; extinction coefficient $\alpha=10^{6} \mathrm{~cm}^{-1}$; specific heat $C=0.72 \mathrm{~J} / \mathrm{g} \mathrm{K}$; ambient temperature $T_{0}=298 \mathrm{~K} ;[\theta(0)=T-$ 298]; $\Delta H_{\mathrm{ev}}=420 \mathrm{~kJ} \mathrm{~mol}^{-1}$

\begin{tabular}{cccccc}
\hline$T$ & $p$ & $j_{\mathrm{ev}}$ & $\Phi_{\mathrm{ev}}$ & $\begin{array}{c}\Phi_{\mathrm{HF}} \\
\left(\mathrm{W} / \mathrm{cm}^{2}\right)\end{array}$ & $\Phi_{\mathrm{ev}} / \Phi_{\mathrm{HF}}$ \\
$(\mathrm{K})$ & $(\mathrm{atm})$ & $\left(\mathrm{mol} / \mathrm{cm}^{2} \mathrm{~s}\right)$ & $\left(\mathrm{cm}^{2}\right)$ & & \\
\hline 2628 & 1.0000 & 0.13 & $6.1 \times 10^{4}$ & $5.7 \times 10^{8}$ & $1.1 \times 10^{-4}$ \\
3000 & 10.8 & 1.32 & $6.3 \times 10^{5}$ & $6.6 \times 10^{8}$ & $9.5 \times 10^{-4}$ \\
3500 & 120 & 13.5 & $6.6 \times 10^{6}$ & $7.8 \times 10^{8}$ & $8.5 \times 10^{-3}$ \\
4000 & 729 & 76.9 & $3.8 \times 10^{4}$ & $9.0 \times 10^{8}$ & $4.2 \times 10^{-4}$ \\
\hline
\end{tabular}

The critical temperature and pressure for silicon have been estimated to be $T_{\mathrm{c}}=4920 \mathrm{~K}$ and $P_{\mathrm{c}}=1450 \mathrm{~atm}[9,10]$.) In Table 2 calculated energy fluxes due to evaporation $\left(\Phi_{\mathrm{ev}}\right)$ and those due to heat conduction $\left(\Phi_{\mathrm{HF}}\right)$ are compared.

Table 2 demonstrates that for silicon up to about $3500 \mathrm{~K}$ evaporation may be neglected with respect to heat conduction.

A more difficult problem is posed for the melting process. Here no direct calculation of the kinetics of melting is available. Experimentally at temperatures considerably above $3500 \mathrm{~K}$ the time constant of melting has been found to be about 1 ps for silicon [7]. However, at temperatures below the boiling point $(2628 \mathrm{~K})$ melting is probably a slow process compared with the rate of heat conduction. If that is correct, then transfer from the condensed phase into the gas phase turns out to be sublimation of a strongly superheated solid. $\Delta H_{\mathrm{ev}}$ in (25) must be replaced by the enthalpy of sublimation $\Delta H_{\text {sub }}$ :

$\Delta H_{\mathrm{sub}}=\Delta H_{\mathrm{ev}}+\Delta H_{\mathrm{m}}$,

where $\Delta H_{\mathrm{m}}$ is the enthalpy of melting $\left(\Delta H_{\mathrm{m}}=50 \mathrm{~kJ} / \mathrm{mol}\right.$ for silicon). Taking this into account $\Phi_{\mathrm{ev}} / \Phi_{\mathrm{HF}}$ is calculated to be 0.013 at $3500 \mathrm{~K}$ and 0.082 at $4000 \mathrm{~K}$.

In the case of high-energy nanosecond pulses, a term for the kinetics of the melting and evaporation processes has to be added to (1).

With ultrashort pulses ( $\sim 100 \mathrm{fs})$ and with fluences above a critical threshold of about $100 \mathrm{~mJ} / \mathrm{cm}^{2}$ [11] it was found that the diamond lattice of silicon becomes unstable; the atoms are displaced by more than $1 \AA$ from their equilibrium positions within less than $200 \mathrm{fs}$. A dense electron-hole plasma is formed $[7,11,12]$ and silicon assumes a metallic character. The equilibration with the lattice occurs with a time constant of about $500 \mathrm{fs}$. Then at very high temperatures far

Table 3. Fluences $\phi_{\max }$ leading to $T_{\max }=3500 \mathrm{~K}$ as a function of $\tau$. Substrate: silicon

\begin{tabular}{|c|c|c|}
\hline $\begin{array}{c}\tau \\
(\mathrm{ps})\end{array}$ & $\begin{array}{l}\text { Half line width } \\
\text { (ps) }\end{array}$ & $\begin{array}{c}(1-R) \phi_{\max } \\
\left(\mathrm{mJ} / \mathrm{cm}^{2}\right)\end{array}$ \\
\hline 0.01 & 0.03 & 9.7 \\
\hline 0.03 & 0.08 & 11 \\
\hline 0.1 & 0.28 & 13 \\
\hline 0.3 & 0.84 & 16 \\
\hline 1 & 2.80 & 20 \\
\hline 3 & 8.39 & 26 \\
\hline
\end{tabular}

above the boiling temperature of silicon fast melting is observed within about 1 ps.

With silicon the fluences $(1-R) \phi_{\max }$ consistent with a maximum surface temperature of $3500 \mathrm{~K}$ are listed in Table 3 as a function of $\tau$. The data are calculated taking into account the temperature dependence of the parameters (thermal diffusivity, specific heat, and density: see Sect. 2.2). At higher energy inputs withdrawal of energy by evaporation cannot be neglected (see Table 4).

\subsection{Heat conduction and Stefan-Boltzmann radiation}

The heat flux of a black body of temperature $T$ is given by:

$\Phi_{\mathrm{SB}}=5.6 \times 10^{-12} T^{4} \mathrm{~W} / \mathrm{cm}^{2}$

This heat flux (in the negative $x$ direction) competes with the flux due to heat conduction (in the positive $x$ direction) as given by (26). With $T_{0} \ll T_{\mathrm{SB}=\mathrm{HF}}$ both heat fluxes are equal for:

$T_{\mathrm{SB}=\mathrm{HF}}=5630 \sqrt[3]{\kappa \varrho \alpha C}$.

Applying (29) to silicon as the substrate we obtain $T_{\mathrm{SB}=\mathrm{HF}}=$ $3.7 \times 10^{5} \mathrm{~K}$. At the highest permissible temperature $T=$ $3500 \mathrm{~K}, \Phi_{\mathrm{SB}}=840 \mathrm{~W} / \mathrm{cm}^{2}$. This is quite negligible compared with the heat flux $\Phi_{\mathrm{HF}}=9.7 \times 10^{8} \mathrm{~W} / \mathrm{cm}^{2}$. We can thus safely neglect Stefan-Boltzmann radiation.

\subsection{Heat conduction and ablation of matter}

If energies higher than those indicated in Table 3 are irradiated ablation of silicon can no longer be neglected. To estimate the amount of matter removed from a silicon surface by evaporation the flux $j_{\mathrm{ev}}$ defined in (24) is integrated over time. Thus the number of moles evaporated from a surface of $1 \mathrm{~cm}^{2}$ area is obtained. The thickness $d$ (in $\mathrm{nm}$ ) of silicon ablated by evaporation is then calculated as:

$d=\frac{10^{7} M}{\varrho} \int_{0}^{\infty} j_{\mathrm{ev}} \mathrm{d} t$

where $M$ is the atomic weight and $\varrho$ the density of the substrate. The amount of energy $\phi_{\mathrm{abl}}$ lost by the probe due to ablation is given by:

$\phi_{\mathrm{abl}}=M \vartheta \int_{0}^{\infty} C\left(T-T_{0}\right) j_{\mathrm{ev}} \mathrm{d} t+\Delta H_{\mathrm{ev}} \int_{0}^{\infty} j_{\mathrm{ev}} \mathrm{d} t$.

Table 4. Thickness $d$ of ablated silicon and energy lost by ablation $\phi_{\mathrm{abl}}$ with $T_{\max }=4920 \mathrm{~K}$

\begin{tabular}{rccrr}
\hline $\begin{array}{c}\tau \\
(\mathrm{ps})\end{array}$ & $\begin{array}{c}\text { Half line width } \\
(\mathrm{ps})\end{array}$ & $\begin{array}{c}(1-R) \phi \\
\left(\mathrm{mJ} / \mathrm{cm}^{2}\right)\end{array}$ & $\begin{array}{c}d \\
(\mathrm{~nm})\end{array}$ & $\begin{array}{c}\phi_{\mathrm{abl}} \\
\left(\mathrm{mJ} / \mathrm{cm}^{2}\right)\end{array}$ \\
\hline 0.1 & 0.28 & 18 & 0.027 & 0.104 \\
0.30 & 0.84 & 22 & 0.061 & 0.25 \\
1 & 2.80 & 28 & 0.16 & 0.63 \\
3 & 8.39 & 36 & 0.41 & 1.6 \\
\hline
\end{tabular}




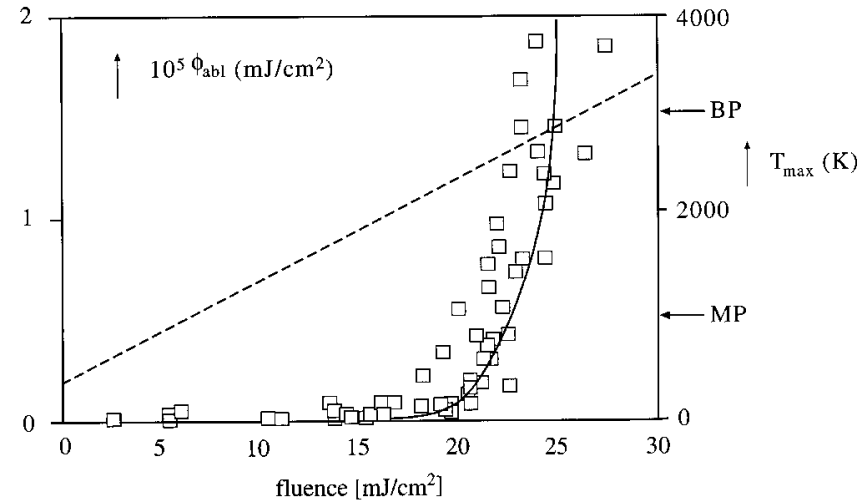

Fig. 3. Ablation of nickel with pulse fluences below the threshold fluence $\phi_{\mathrm{T}}$. Measured ablation of nickel in relative units (squares) [13]. Calculated ablation (drawn out curve). $\varrho=8.90 \mathrm{~g} / \mathrm{cm}^{3}, C=0.44 \mathrm{~J} / \mathrm{gk}, R=0.475$, $\kappa=0.24 \mathrm{~cm}^{2} / \mathrm{s}, \Delta H_{\mathrm{v}}=6.3 \mathrm{~kJ} / \mathrm{g}, \alpha=1.25 \times 10^{6} \mathrm{~cm}^{-1}$, boiling point $=$ $3187 \mathrm{~K}$. Dashed curve: $T_{\max }=$ maximum temperature of surface as function of fluence

In Table 4 the amount of evaporated silicon in terms of $d$ and $\phi_{\mathrm{abl}}$ are listed as a function of the half line width of the laser pulse under conditions that the maximum temperature is $4920 \mathrm{~K}$ (the critical temperature of silicon [9]). As in Table 3 the data are calculated taking into account the temperature dependence of the parameters. (The influence of the temperature dependence of the parameters on calculated values of $d$ and $\phi_{\mathrm{abl}}$ is rather strong. Calculating $d$ and $\phi_{\mathrm{abl}}$ assuming constant values of $\kappa, \varrho$, and $C$ leads to values smaller than the data of Table 4 by about a factor of 10.)

Using a laser ionization time-of-flight mass spectrometer Preuss et al. [13] observed after irradiation of a nickel probe with $0.5 \mathrm{ps}$ laser pulses $(\lambda=248 \mathrm{~nm})$ that ablation already occurs at $\phi=20 \mathrm{~mJ} / \mathrm{cm}^{2}$ considerably below the threshold fluence $\phi_{\mathrm{T}}=85 \mathrm{~mJ} / \mathrm{cm}^{2}$ [14]. In Fig. 3 calculated values for ablation in terms of $\phi_{\mathrm{abl}}$ using (31) are shown and compared with experimental values [13]. The latter are only available on a relative scale. Therefore the experimental data have been scaled to match the calculated ones.

Notwithstanding this scaling, both calculated and experimental data demonstrate the existence of a lower threshold of $20 \mathrm{~mJ} / \mathrm{cm}^{2}$ for measurable evaporation of nickel according to (31). The amount of evaporated nickel is very small. Even after the application of 1000 pulses to the same location of the nickel probe no change of the surface was detectable [13]. This is in accordance with (30): for $\phi=30 \mathrm{~mJ} / \mathrm{cm}^{2}$ and 1000 pulses the depth of ablated nickel is calculated to be only $0.03 \mathrm{~nm}$.

Increasing fluences above a second threshold fluence ( $\phi_{\mathrm{T}}=85 \mathrm{~mJ} / \mathrm{cm}^{2}$ for $\left.\mathrm{Ni}[14]\right)$ leads to temperatures above the critical temperature $\left(T_{\mathrm{c}}=5700 \mathrm{~K}\right.$ for nickel [10]) and the rate of ablation will drastically increase. At these high temperatures the change of liquid nickel towards supercritical nickel gas will be very fast and heat conduction cannot remove the energy fast enough. Due to thermal expansion and the finite velocity of sound the probe is under high pressure. So we expect expulsion of that part of nickel that is heated above the critical temperature. Under these conditions a reliable calculation of $\theta(x, t)$ is no longer possible.

At the lower threshold $\left(20 \mathrm{~mJ} / \mathrm{cm}^{2}\right)$ evaporation from the surface, and at the higher threshold $\left(85 \mathrm{~mJ} / \mathrm{cm}^{2}\right)$ ejection from the bulk of nickel, begins to be observable.

Acknowledgements. The author wishes to thank Prof G. Marowsky for useful discussions.

\section{References}

1. J.F. Ready: J. Appl. Phys. 36, 462 (1965)

2. J.F. Ready: Effects of High-Power Laser Radiation (Academic Press, New York 1971)

3. D. Bäuerle: Laser Processing and Chemistry 2.Ed. (Springer, Heidelberg 1996)

4. H.R. Shanks, P.D. Maycock, P.M. Sidles, G.C. Danielson: Phys. Rev. 130, 1743 (1963)

5. International Critical Tables Vol.1; page 104, (McGraw-Hill, New York 1933)

6. K. Mitra, S. Kumar, A. Vedavarz: J. Appl. Phys. 80, 675 (1996)

7. H.W.K. Tom, G.D. Aumiller, C.H. Brito-Cruz: Phys. Rev. Lett. 60, 1438 (1988)

8. H. Hertz: Ann. Physik (Leipzig) 17, 177 (1882);

M. Knudsen: Ann. Physik (Leipzig) 42, 697 (1915)

9. Gmelins Handbuch der Anorganischen Chemie; Silicium Teil B, System 15, page 62 (Verlag Chemie, Weinheim 1959)

10. R.Fürth: Proc. Roy. Soc. A 183, 87 (1945)

11. C.V. Shank, R. Yen, C. Hirlimann: Phys. Rev. Lett 51, 900 (1983)

12. P. Stampfli, K.H. Bennemann: Appl. Phys. A 60, 191 (1995)

13. S. Preuss, E. Matthias, M. Stuke: Appl. Phys. A 59, 79 (1994)

14. S. Preuss, A. Demchuk, M. Stuke: Appl. Phys. A 61, 33 (1995) 